\title{
Cuba : mutations sociales et défis politiques
}

Janette Habel

\section{(Q) OpenEdition \\ Journals}

Édition électronique

URL : http://journals.openedition.org/plc/458

DOI : $10.4000 /$ plc. 458

ISSN : 2117-5209

\section{Éditeur}

L'Harmattan

\section{Édition imprimée}

Date de publication : 1 janvier 1999

Pagination : 51-67

ISSN : 1279-8657

\section{Référence électronique}

Janette Habel, «Cuba : mutations sociales et défis politiques », Pouvoirs dans la Caraïbe [En ligne],

11 | 1999, mis en ligne le 14 mars 2011, consulté le 19 avril 2019. URL : http://

journals.openedition.org/plc/458 ; DOI : 10.4000/plc.458 


\section{Cuba : mutations sociales et défis politiques}

par Janette HABEL Maître de conférences Université de Marne-la-Vallée Institut des Hautes Etudes d'Amérique latine

\section{LES RAISONS D’UNE RESISTANCE}

«Will Castro fall ? »s'interrogeait-on aux Etats-Unis après la chute du mur de Berlin. «L'an prochain à La Havane » assurait-on à Miami... Au début des années 1990 après la chute de l'URSS, Cuba semblait totalement isolée sur le plan international. A l'instar de nombreux observateurs Ronald Linden affirmait que «sans changement du cours suivi par les Etats de l'Europe de l'est ou sans changements à Cuba même, cet isolement continuerait $»^{1}$. Les quelques années écoulées depuis ont infirmé ce jugement.

A force de ne voir dans le castrisme qu'un goulag tropical, les médias ont fait du régime cubain un épouvantail totalitaire dont seule la répression politique à l'égard de ses opposants expliquerait la survie.

S'agissant de Cuba, il faut donc se méfier des pronostics. Comment expliquer cette résistance ? Par la stratégie de la direction cubaine? Si l'on en croit Fidel Castro, c'est la fermeté des principes qui a permis de «tenir». Mais la réalité des faits et des politiques suivies montre que certains de ces « principes » sont mis à mal.

1. C. Mesa-Lago, Cuba after the Cold War, University of Pittsburgh, 1993, p. 51. 
L'appui populaire dont a bénéficié la Révolution Cubaine s'explique par les deux composantes qui l'ont inspirée : la défense de la souveraineté nationale et la justice sociale. Le sentiment national reste aujourd'hui vivace bien que chez certains intellectuels et dans la jeunesse on minimise son importance en privilégiant l'existence d'une cubanité dont l'île n'aurait pas le monopole. On ne parle plus des «gusanos» (les «vers de terre », nom donné aux «traitres » qui partaient au début de la révolution) et nécessité oblige, on organise des conférences sur la Nation et l'Emigration. Mais on continue à craindre l'hégémonisme du Nord « revuelto y brutal » comme disait José Martí. Le souvenir de l'oppression et des humiliations subies au $\mathrm{XX}^{\text {ème }}$ siècle (dont la base de Guantánamo est un symbole) donnent au discours castriste une légitimité qui n'est pas factice, dans une population majoritairement noire moins sensible aux attraits de l'« American way of life ». Le renforcement de l'embargo nord-américain depuis la crise avec les lois Torricelli et Helms-Burton ne peut que conforter cette défiance.

Cependant les conquêtes sociales sont mises à mal par dix ans de période spéciale, de réformes économiques marchandes. L'austérité des uns contraste avec l'enrichissement des autres. La réflexion et les débats politiques sont figés, laissant le champ libre à la parole d'une Eglise catholique pourtant minoritaire. Le marché encadré par l'ordre étatique, objectif avoué de certains hiérarques, produit déjà ses effets désagrégateurs.

\section{RAVAGES DU MARCHE ET FRACTURE SOCIALE}

Les bouleversements économiques qui affectent le pays depuis presque dix ans - la «période spéciale » consécutive à l'effondrement de l'URSS a été décrétée en août 1990 - ont profondément ébranlé la stabilité de l'île. Le processus de libéralisation économique cubaine a été rapide si l'on tient compte du fait qu'il n'a vraiment commencé qu'il y a cinq ans, en 1993. Au delà des différences de situation, rappelons pour mémoire que l'ouverture économique en Chine date de 1978 et au Viêtnam de 1986. 
Pour faire face à la crise, le gouvernement cubain a combiné ouverture économique et centralisation autoritaire sans offrir néanmoins de perspective stratégique à long terme. Bénéfiques pour certains secteurs de l'économie et secondairement pour l'appareil du Parti, les réformes adoptées ont provoqué une dislocation idéologique et sociale profonde. De nouveaux acteurs sont apparus qui suscitent la protestation des laissés pour compte de l'ordre antérieur. "Le secteur vert - celui des dollars - progresse, il permet chaque fois plus de choses, il envahit les boutiques et d'autres domaines. Mais le salaire, l'aide sociale diminuent et si on me paye en pesos, qu'est ce que je vais devenir avec mes pesos face à ceux qui ont accès aux dollars ? ». Cette interrogation ${ }^{2}$ d'un Havanais reflète bien le fossé que crée dans la société cette pyramide inversée qui place au bas de la hiérarchie sociale les anciens cadres privilégiés du régime, médecins, ingénieurs, professeurs, qui gagnent moins que des travailleurs non qualifiés.

Il existe désormais des enfants ou des vieillards sous-alimentés parmi les catégories les plus défavorisées de la population, alors que des fortunes personnelles s'accumulent par ailleurs. Au cours de l'enquête menée par Guillermo Milán, chercheur à l'Institut de Philosophie de La Havane, cette polarisation sociale apparaît nettement. Si $15 \%$ des personnes interrogées se considèrent comme extrêmement nécessiteuses, $4 \%$ estiment que tous leurs besoins sont satisfaits. Parmi les autres personnes interviewées, ce sont les problèmes économiques qui sont le plus souvent mentionnés : les bas salaires, le coût élevé de la vie, les problèmes de transport et de logement. Ces indications sont corroborées par la tendance à la concentration des dépôts dans les Caisses d'Epargne : les dépôts des gros épargnants ont augmenté alors que ceux des petits ont diminué (les soldes supérieurs à 10000 pesos représentaient $36 \%$ de l'ensemble en 1994 et $43 \%$ en 1996, alors que les soldes inférieurs à 2000 pesos sont passés pendant la même période de $22 \%$ à $15 \%)^{3}$.

2. Manuel Vasquez Montalbán, Y Dios entró en La Habana, Ed. El Pais Aguilar, 1998, Madrid, p. 29.

3. CEPAL, La economía cubana, Fondo de Cultura Económica, México, 1997. 
Dans les années 1980, $95 \%$ de la population active était employée par l'Etat. On estime aujourd'hui à 20 ou $25 \%$ selon les auteurs ${ }^{4}$ la proportion de la force de travail employée dans le secteur privé légal ou informel depuis les réformes des années 1990. Louer une chambre, vendre des pizzas ou du café dans les rues, permet d'arrondir les fins de mois. Plus rentables sont les petits restaurants privés ou les réparations domestiques ou mécaniques effectuées par des ouvriers qualifiés. Enfin les revenus apportés par la prostitution sont parfois considérables. Dans ces conditions, à quoi servent un salaire ou des diplômes ? Il devient prioritaire de rétablir une certaine logique dans la hiérarchie des salaires et des revenus.

Quant au plein emploi il n'est plus qu'un souvenir : l'emploi public s'est réduit de $15 \%$ entre 1993 et $1996^{5}$. Des analystes de la CEPAL estiment que le chômage - sous-emploi et chômage déclaré calculés sur la base des niveaux de productivité de 1989 - atteignait $34,1 \%$ en $1996^{6}$ ce qui équivaut à la nécessité de créer des emplois pour plus d'un million de personnes. Un problème aggravé par l'écart entre le niveau de qualification existant et les propositions d'emploi disponibles d'une part, et par la faible disponibilité de logements qui limite la mobilité de la main-d'œuvre d'autre part.

C'est dans ce fossé entre les principes proclamés par le régime et la réalité quotidienne que s'infiltrent de nouvelles «valeurs » mercantiles ou spirituelles.

\section{L'IDEOLOGIE DE L'EGLISE EN PROGRES}

Le voyage du Pape a permis d'élargir les marges de manœuvre de l'Eglise catholique pourtant traditionnellement minoritaire. Pour la

4. Cf. Nacla, Mars-avril 1999, p. 18 et 24, La revue Temas $\left(\mathrm{n}^{\circ} 11\right.$, p. 42) l'estime à $22 \%$ en 1994.

5. H. Escaith, « Cuba pendant la "période spéciale" : ajustement ou transition ? », à paraître dans les Cahiers des Amériques latines, 1999.

6. CEPAL, La economía cubana, Fondo de Cultura Económica, México, 1997, p. 188. 
première fois un contre-pouvoir existe, dont l'autonomie et l'influence internationale n'ont pas d'équivalent. Pour la première fois, les Cubains ont entendu dans les médias un discours - celui de Jean-Paul II mais aussi (surtout) celui de $\mathrm{P}$. Meurice Estiù Archevêque de Santiago de Cuba - critiquant publiquement le système de Parti unique. Or non seulement cette contestation politique est restée sans réponse mais l'Eglise apparaît désormais plus crédible que la dissidence qui n'a jamais eu de programme à long terme. Elle incarne une cohérence idéologique, sociale, économique différente du régime. Elle tente, à la différence de la dissidence, de ne pas laisser le monopole du nationalisme au pouvoir en s'opposant avec fermeté à l'embargo américain tout en dénonçant les méfaits du néolibéralisme. Elle préconise la réconciliation nationale tout en critiquant les extrémistes de Miami. Elle met à profit le scepticisme qui affecte le marxisme officiel depuis l'effondrement du socialisme réel en offrant une «spiritualité » dont l'absence apparaît comme un des grands échecs du régime.

Sur le plan social la hiérarchie catholique insiste sur la nécessité de reconstruire une société civile. L'Evêché de Pinar del Rio s'emploie avec succès à animer des «Cercles de formation civiques », réunions de débat et d'échanges. L'Eglise cubaine donne un certain appui aux syndicats chrétiens indépendants par le biais de la CLAT (Confédération Latino-américaine des Travailleurs). Le secrétaire général d'un petit groupe de syndicalistes, Pedro Pablo Alvarez Ramos, invité au $11^{\text {ème }}$ congrès de la CLAT en novembre 1998 à México fut autorisé à y participer. Cependant la CISL s'inquiète dans son bulletin de l'existence possible dans les zones franches de « maquiladoras ${ }^{7}$ à $\mathrm{Cuba}$, « de la répression anti-syndicale, du problème posé par le système de paiement en dollars des salaires dans les entreprises mixtes et de l'embauche préférentielle » tout en dénonçant l'incapacité du syndicat officiel, la CTC, «d'abandonner son rôle de complice du pouvoir ».

Enfin Caritas bénéficie de l'aide et des dons des Eglises étrangères en premier lieu de celles des Etats-Unis, d'Allemagne, ou

7. Usines d'assemblage, sous-traitance. 
du Canada. L'assistance apportée aux plus pauvres dans les paroisses, en renforçant le sentiment d'appartenance à une communauté solidaire, a accru le prestige du clergé investi dans les tâches humanitaires. Les prêtres insistent sur la nécessité de revaloriser la famille. Ils sont écoutés, car l'échec des «écoles à la campagne » où les carences de l'encadrement scolaire ont provoqué des difficultés relationnelles chez les adolescent(e)s, l'absence de repères et l'instabilité familiale en cette période de crise préoccupent les parents et les éducateurs.

Sur le plan économique, les propositions faites sont empreintes d'une grande prudence. Elles présentent cependant l'initiative privée et le marché comme étant plus efficaces voire plus démocratiques que l'économie de commandement désormais perçue comme un échec.

Si l'on ajoute à l'ensemble de ce dispositif les relais internationaux dont dispose le Vatican, on comprend l'espace potentiel que peut occuper la seule institution indépendante du pouvoir.

\section{LA NOMENKLATURA ET \\ LES « CAMARADES INVESTISSEURS » (H. DILLA $\left.{ }^{8}\right)$}

La décentralisation et la réforme des entreprises donnent un pouvoir important aux gérants d'entreprises et aux directeurs : si l'on en croit Vasquez Montalbán ${ }^{9}$, Eusebio Leal - historiador de la Ciudad et responsable de l'aménagement de la vieille Havane - a depuis 1994 les mains libres pour faire et défaire ce qu'il veut dans le centre historique de la capitale, «ce qui d'après ses amis et ses ennemis en a fait le premier entrepreneur de Cuba de par les affaires qu'il a développées et dont les bénéfices servent à restaurer la ville ${ }^{10}$.

La bureaucratie d'Etat n'est pas homogène mais elle est sous contrôle. Fidel Castro garde la main mise absolue sur l'appareil d'Etat

8. Haroldo Dilla, «Comrades and investors : the uncertain transition in Cuba », Socialist Register, 1999.

9. Manuel Vasquez Montalbán, Y Dios entró en La Habana, Ed. El Pais Aguilar, Madrid, 1998.

10. Manuel Vasquez Montalban, Ibid. 
même si la gestion de la politique économique lui échappe en partie. Il a la capacité de concilier des intérêts contradictoires entre le personnel de gestion dont les compétences économiques sont nécessaires et le personnel d'encadrement moins compétent mais qui assure un contrôle politique indispensable. L'une des difficultés politiques induites par les réformes économiques tient à la restructuration des entreprises d'Etat et des ministères dans la mesure où elle implique une rationalisation des effectifs et des compétences (ou des incompétences) susceptibles de provoquer des oppositions durables chez les cadres administratifs. Ces derniers ne sont favorables aux réformes que dans la mesure où elles ne compromettent pas leur statut social. Entre les «camarades investisseurs ${ }^{11}$ qui veulent généraliser leurs succès économiques et les camarades conservateurs chargés d'assurer la stabilité du pouvoir politique, l'harmonie est provisoire.

Au delà des différences, l'hostilité rencontrée par le premier Ministre Zhu Rongji en Chine, dont le programme prévoit la diminution de $50 \%$ des cadres des entreprises d'Etat ${ }^{12}$ (remettant en cause le «bol de riz en fer» la garantie de l'emploi) fait réfléchir les responsables cubains qui suivent de près - bien qu'ils s'en défendent l'expérience chinoise. L'instabilité sociale est un problème crucial pour un pays qui ne dispose pas, il s'en faut de beaucoup, des ressources de l'empire du Milieu. L'une des incertitudes de l'aprèscastrisme tient à la capacité du successeur désigné, Raoul Castro d'assurer l'avenir du régime. Certes le Ministre des Forces Armées est aussi le deuxième secrétaire du Parti et l'imbrication des deux institutions est grande surtout au sommet de l'Etat : six militaires sont membres du Bureau Politique (le quart de ses membres) mais si l'armée est fortement investie sur le plan économique, c'est le PCC qui a en charge l'appareil administratif national, provincial et municipal, et les organisations de masse qui encadrent l'ensemble de la population.

Or la réalité militante du parti, sa vie interne et sa cohésion se sont considérablement affaiblies avec la crise.

11. Haroldo Dilla, «Comrades and investors : the uncertain transition in Cuba », Socialist Register, 1999.

12. International Herald Tribune, 17/18 avril 1999. 
Les dirigeants cubains ont toujours justifié par l'agression américaine et la volonté annexionniste cachée de Washington l'existence d'un parti unique comme seule garantie d'unité et de cohésion nationales. En 1997 évoquant l'Afrique et ses problèmes d'unité nationale, Fidel Castro déclarait : «Les pays sont aujourd'hui plongés dans le chaos, ils sont divisés en de nombreuses fractions, en de nombreux partis, c'est ce qu'on a exigé d'eux. En Afrique même les tribus ont été transformées en partis. Nous, nous avons fait le contraire : nous avons transformé les partis en une tribu, c'est à dire en une seule famille $»^{13}$. Justifiant ainsi au nom de la résistance nationale le monolithisme interne au Parti lui-même, le patriarche conduit à son inéluctable affaiblissement.

Certes on ne saurait sous-estimer les difficultés de construction d'un Etat-île-nation à proximité de la première puissance impériale dont la Présidence est soumise au puissant lobby de la bourgeoisie cubaine exilée. Mais comme le constate María López Vigil, observatrice attentive de la réalité cubaine et directrice de la revue centroaméricaine ENVIO, les conséquences de la symbiose parti-Etat sont de plus en plus négatives. Elle rapporte ces propos : «Le Parti en ces dernières années s'est réduit à une direction au sommet qui a un lien direct avec l'Etat. C'est ce groupe dirigeant (dirigencia cupular) qui conduit et oriente les changements qui ont lieu à Cuba et qui affectent le portefeuille, l'esprit et l'âme de toute la société ». «Qui affectent ma vie, tu comprends, et je n'en ai qu'une seule » proteste son interlocuteur. Il regrette qu'un grand nombre de militants du Parti, étrangers à ce groupe dirigeant, attendent: «ils sont capables, ils veulent participer, travailler et faire part de leurs opinions, mais voyant qu'ils ne peuvent rien faire, rien changer, et qu'ils n'ont aucune influence ils s'aigrissent. Et deviennent indisciplinés. Au delà de ces militants il y a la masse de la population qui n'est pas militante mais qui reste révolutionnaire, des Cubains et des Cubaines qui veulent, peuvent et doivent participer et qui sont aussi dans l'expectative $\gg 14$.

13. Cité par María López Vigil, Revue Envío, n 184, juillet 1997, Managua. 14. Ibid. 
« Au fur et à mesure que se construisait le Parti unique, auquel très peu d'intellectuels eurent accès, celui-ci commença à assumer le rôle d'un cerveau unique qui décidait pour tout le monde, qui effectuait tous les choix. Le parti unique fut l'un des éléments les plus destructeurs de ce lien entre les intellectuels et le processus historique qui avait lieu dans le pays » écrit l'essayiste cubain Lisandro Otero qui vit aujourd'hui au Mexique ${ }^{15}$ et se déclare partisan d'un «socialisme efficace $»$.

L'Armée pourrait-elle être un relais politique fiable en cas de crise ? Le pouvoir a su gérer jusqu'alors les tensions qui auraient pu surgir de deux phénomènes concomitants: le retour des cinquante mille soldats cubains d'Angola d'une part (après la signature de l'accord Tripartite entre l'Angola, Cuba et l'Afrique du Sud en décembre 1988) et la reconversion économique d'une partie des militaires privés de vocation internationale et soumis à des restrictions budgétaires sans précédent impliquant la diminution de ses effectifs d'autre part. Alors que l'armée cubaine occupait la deuxième place en Amérique latine après le Brésil, en 1995 ses effectifs avaient diminué de moitié environ par rapport à $1990^{16}$.

L'exemple des entreprises gérées par l'armée était depuis longtemps donné en exemple. Les entreprises dépendant du MINFAR avaient obtenu pour la seule année 1987 une augmentation de $27 \%$ de la productivité, en flexibilisant l'organisation de la production et la fixation des salaires ${ }^{17}$.

C'est ce rôle pilote utilisant des critères de rentabilité capitalistes dans la gestion de ses entreprises qui a facilité la reconversion des officiers dans les joint ventures et l'investissement d'une partie des militaires dans les activités agricoles ou touristiques dépendant du MINFAR, les plus anciens partant à la retraite. « Mas vale frijoles que

15. La Vanguardia, 19 avril 1999, Mexico.

16. Phyllis Greene Walker, "Cuba's revolutionary armed forces: adapting in the new environment », Cuban Studies, ${ }^{\circ}$ 26, University of Pittsburgh, p. 61.

17. H. Escaith, «Cuba pendant la "période spéciale" : ajustement ou transition? », à paraître dans les Cahiers de l'Amérique latine, 1999. 
cañones » (il vaut mieux produire des haricots que fabriquer des canons) avait déclaré Raoul Castro en avril $1993^{18}$.

Sur les marchés agricoles ou lors de la zafra, les militaires sont omniprésents. Dirigée par un général vice-ministre des FAR et membre du Bureau politique, l'entreprise Gaviota gère des hôtels, des entreprises mixtes, des plages, des centres de loisirs. Au Bureau Politique, ils détiennent des postes clés : le sucre, les transports, les communications...

Cette réinsertion n'est cependant pas générale : certains officiers sont restés à l'écart et il n'est pas rare de rencontrer tel capitaine de l'Armée Rebelle faisant office de chauffeur de taxi, voire même un Commandant de la Sierra Maestra obligé de louer une chambre dans sa maison pour gagner quelques dollars. Mais comment ce nouveau rôle économique va-t-il modifier la conscience de la grande muette ? L'armée cubaine est issue de l'Armée Rebelle et nombre de ses cadres sont encore vivants, à commencer par son fondateur. Elle a été jusqu'alors fidèle aux idéaux de la lutte insurrectionnelle mais beaucoup de ses cadres ont été formés à l'école soviétique. Mais ses fonctions sont aujourd'hui tout autres et ces nouveaux gestionnaires trouvent leur fierté dans la rentabilité et l'efficacité économique obtenues grâce à une discipline militaire qui n'est pas généralisable à toute la société quelle qu'en soit la tentation.

Pourtant, dans l'ensemble, l'Armée est apparue comme le promoteur des réformes économiques, comme une institution stable et professionnelle, efficace, ayant fait preuve d'une grande capacité d'adaptation aux nouvelles conditions sans vouloir jouer un rôle politique.

\section{VOUS AVEZ DIT SOCIETE CIVILE?}

Le concept de société civile avait été évoqué par F. Castro en 1992, mais il n'est apparu dans un texte officiel à Cuba qu'en 1996

18. El Sol (Mexico), 21 avril 1993. 
dans un rapport très polémique de Raoul Castro prononcé au nom du Bureau Politique. Le Deuxième Secrétaire du Parti et Ministre des Forces Armées mettait en cause les centres de recherche, en particulier le Centre d'Etudes sur l'Amérique $\left(\mathrm{CEA}^{19}\right)$, et certaines ONG accusés de former une cinquième colonne sous influence américaine et de faciliter ainsi l'application du «track two ( carril $\operatorname{dos}^{20}$ ), en contribuant à saper de l'intérieur l'unité nationale au profit des intérêts américains.

En octobre 1997, le $\mathrm{V}^{\text {ème }}$ Congrès du PCC allait rectifier cette analyse : le thème de la société civile était mentionné sous une forme positive... assorti de l'adjectif «socialiste», témoignant comme le constate le chercheur cubain Haroldo Dilla ${ }^{21}$ de «la volonté de la bureaucratie de contrôler la société civile, de décider de qui en fait partie et de qui en est exclu et d'exercer ainsi une sorte de contrôle administratif sur son évolution $\gg$.

Les craintes de voir émerger des secteurs de la société échappant au contrôle du pouvoir ne datent pas de la crise. L'un des traits marquants du système cubain est d'avoir contribué de façon exceptionnelle à la promotion sociale de ses citoyens, en élevant leur qualification professionnelle, pour bloquer ensuite leur épanouissement par un contrôle politique et social sévère. La crise économique a aggravé le phénomène dès lors que sont apparus de nouveaux acteurs sociaux établis à leur compte, légalement ou dans le secteur informel, échappant à la tutelle de l'Etat ${ }^{22}$.

19. Le Centre d'Etudes sur l'Amérique regroupait de nombreux chercheurs et publiait une revue - «Cuadernos de Nuestra America » - appréciée pour le sérieux de ses articles et la qualité de ses analyses critiques. La revue n'est plus reparue depuis 1996 et les principaux chercheurs du centre, le directeur et le directeur adjoint ont été dispersés dans d'autres institutions.

20. La diplomatie américaine favorise désormais les contacts « de peuple à peuple » comme tactique subversive plus efficace que la confrontation.

21. Haroldo Dilla, «The virtues and misfortunes of civil society», NACLA, Report on the Americas, $\mathrm{n}^{\circ}$ 5, mars/avril 1999.

22. Chercheur au CEA Hugo Azcuy est mort d'un infarctus en mars 1996 après le discours accusateur de Raoul Castro. 
En d'autres termes, comme l'affirmait le chercheur Hugo Azcuy "la nécessité d'une expression plurielle de la société cubaine s'est accentuée au cours des dernières années de crise» et «la reconnaissance d'un espace social autonome doit être mise en rapport à Cuba avec la perte relative de la part de l'Etat de sa capacité de satisfaire entièrement les besoins de la population, et d'autre part avec l'apparition d'une économie privée $»^{23}$.

Le « rôle recteur » de l'Etat et du Parti unique se heurtent ainsi à une contradiction majeure : en refusant d'accepter cet espace social autonome, le pouvoir se prive des moyens démocratiques de contrebalancer les effets des réformes marchandes. Celles-ci combinées au monopole du parti, engendrent corruption et délinquance, alors que n'existent ni transparence ni mécanismes de contrôles et que le secret bureaucratique protège les fraudeurs. Il ne reste au gouvernement que le recours à la répression pour lutter contre la délinquance, les délits, les vols dans le secteur d'Etat.

Souvent analysée comme un symbole de décomposition et une perversion immanente, la corruption est d'abord le résultat de la dollarisation, de l'autonomie croissante laissée aux «managers ». A une échelle plus limitée elle est l'expression des stratégies de survie utilisées par la population : marché noir, prostitution ou délinquance. Si les discours officiels - en particulier ceux de Fidel Castro - font état des difficultés du pays, ils restent vagues et imprécis concernant les souffrances des secteurs les plus fragilisés de la population. La façon dont le Commandant en Chef a évoqué la prostitution comme le produit d'une coquetterie féminine est révélatrice : «Personne ne peut prétendre être dans la détresse ici et une paire de chaussures à talons hauts, un soulier de luxe, un parfum séduisant, un nouveau vêtement ne peuvent compromettre l'honneur et la survie d'une nation $»^{24}$. L'impossibilité de s'alimenter plus de deux semaines (dans le meilleur des cas et mal de surcroit) avec la libreta (carnet de rationnement), l'existence d'un sous-emploi massif et la déqualification professionnelle font que les catégories les plus vulnérables de la population

23. Temas, ${ }^{\circ}$ 4, La Havane, 1995.

24. Fidel Castro, Granma, 8 janvier 1999. 
connaissent une grande pauvreté : c'est le cas des vieillards lorsqu'ils ne bénéficient pas de soutiens familiaux, des mères célibataires avec des enfants, des noirs qui ne reçoivent pas de remesas de l'étranger car l'exil est majoritairement blanc, des chômeurs qui ne disposent que de $60 \%$ de leur salaire (en pesos), ou de ceux qui se trouvent dans des régions où les retombées du tourisme ne se font pas sentir. Le maintien - mais aussi la dégradation - des services sociaux gratuits ne suffit pas à éviter la misère. On assiste bien dans certains cas à la destruction du tissu social antérieur. Il y a désormais dans la crise des gagnants et des perdants, même si le régime cherche encore à protéger ces derniers.

Le viol des normes sociales, les suicides, l'émigration sont la conséquence de cette situation. Face au désarroi et à la démoralisation, certains chercheurs évoquent une désorientation sociale voire des phénomènes d'anomie ${ }^{25}$. Le journal de la CTC «Trabajadores» a exprimé sa préoccupation face à la corruption croissante et la démotivation des salariés ${ }^{26}$.

Comme dans toutes les crises sociales, la jeunesse, les artistes, les intellectuels réfractent ces tensions. En 1998 un nouvel incident est venu ébranler la confiance des milieux artistiques après que le Commandant en Chef a critiqué le film Guantanamera, œuvre du célèbre réalisateur Tomas Gutierrez Alea mort depuis deux ans, tout en avouant ne pas avoir vu le film concerné. «Quelle est cette île triste et noire ? »s'interrogea alors l'écrivain Abilio Estevez ${ }^{27}$.

Bien que toujours faible, la dissidence se montre plus audacieuse depuis le voyage du Pape. Des rassemblements protestataires ont eu lieu lors de plusieurs procès. Les participants étaient peu nombreux mais les répercussions plus importantes que par le passé. Publiée en février 1999, la «Loi de protection de l'indépendance nationale et de l'économie cubaine » vise entre autres à limiter l'expression politique

25. D'après une enquête de l'Institut de Philosophie $30 \%$ des personnes interrogées pensent que le capitalisme est une alternative sociale qui leur permettrait de s'accomplir en tant qu'individus (Nacla mars/avril 1999, p. 35).

26. Trabajadores, $\mathrm{n}^{\circ}$ 23, Mai/juin 1998.

27. Auteur de Tuyo es el reino (Ce royaume t'appartient) paru en France aux éditions Grasset, 1999. 
publique des journalistes indépendants. Le procès de quatre dirigeants ${ }^{28}$ du Groupe de Travail de la Dissidence Interne emprisonnés depuis juillet 1997 a confirmé le renforcement de la répression. Accusés par le procureur d'atteinte à la sécurité de l'Etat et de sédition, les quatre sont considérés coupables d'avoir servi les intérêts des EtatsUnis, encouragé l'abstention électorale et voulu faire obstacle à la tenue $\mathrm{du} \mathrm{V}^{\mathrm{ème}}$ congrès du PCC et à la visite de Jean-Paul II. Qualifiés par le journal Granma de véritables « traîtres à la nation, au peuple et à ses valeurs », l'éditorialiste souligne que « pour mener à bien ses plans de subversion interne les Etats-Unis paient des agents, organisent et financent des groupes, encouragent des leaders qui ne sont connus qu'à l'extérieur mais totalement ignorés dans le pays ${ }^{29}$.

Il est vrai que les «quatre» ont appelé les entrepreneurs étrangers à ne pas investir à Cuba. Ce faisant, ils sapent la possibilité de gagner un appui populaire et montrent les limites d'une dissidence incapable de défier Fidel Castro sur le terrain de la souveraineté nationale. Mais on ne peut assimiler ces appels à une atteinte à la sûreté de l'Etat. Et que penser d'un pouvoir qui contrôle tous les médias et ne peut riposter? L'argument des dirigeants selon lesquels le pays est en guerre et ne peut se permettre le luxe d'un débat n'est pas recevable. Aussi grave soit-il - et il ne saurait être question ici de le minimiser - l'embargo ce n'est ni la guerre ni un «génocide », terme employé par F. Castro. Ne faut-il pas plutôt chercher dans les dysfonctionnements structurels de l'appareil d'Etat et dans l'impasse politique où se trouve la direction cubaine l'explication de ses choix autoritaires?

\section{QUEL PROJET A LONG TERME?}

Quand on demande aux responsables cubains quel est leur programme, leur projet à long terme, les réponses sont évasives. Interrogé en 1993 lors d'un séminaire organisé par l'IRELA (Instituto

28. Wladimiro Roca, Marta Beatriz Roque, Felix Bonne, René Gomez Manzano.

29. Cuban Review, avril 1999, p. 1. 
de Relaciones Europeo - latinoamericanas - dont le siège est à Madrid), Carlos Lage s'était livré à une explication alambiquée et peu convainquante ${ }^{30}$ :

«On dit beaucoup que nous n'avons pas de programme, de nombreux amis nous le disent, beaucoup de personnes expriment cette préoccupation et nous devons réfléchir sur ces opinions, nous devons tenir compte de ces critères, nous sommes d'accord pour en prendre acte et nous le faisons. Je dis cependant que nous aspirons à un système socialiste, c'est à dire un système où la production soit propriété étatique, c'est à dire un système d'égalité sociale, de justice sociale. Dans ce système socialiste, dans les conditions où nous devons le développer aujourd'hui, nous devons concevoir, promouvoir car nous l'estimons utile (conveniente) une participation croissante du capital privé, principalement en association avec la propriété d'Etat socialiste, en recherchant de surcroît la technologie et les marchés ; la pratique de ces dernières années nous a montré que c'était possible. Dans ce système socialiste nous devons donner une place importante à la production coopérative, essentiellement dans l'agriculture, pour stimuler et motiver davantage la participation de tous ceux qui sont intéressés à la production ».

Quant à Fidel Castro comment voit-il le moment présent ? De ses différents discours on peut déduire qu'il analyse la période actuelle comme un recul négatif mais nécessaire compte tenu des rapports de force mondiaux. «Le monde est un gigantesque casino, le monde est un chaos » a-t-il déclaré lors de la réunion de l'Association des Etats de la Caraïbe (AEC) à St Domingue en avril 1999 ${ }^{31}$. Au cours de cette marche arrière, il faut préserver les conquêtes essentielles de la Révolution - pour lui, le pouvoir politique - en attendant des jours meilleurs permettant de reprendre la construction du socialisme. Sans doute n'avait-il pas prévu au début des années 1990 que la crise serait aussi longue et ses ravages aussi profonds alors qu'il entre dans sa soixante treizième année. De quoi sera fait l'après-castrisme ? Un leadership sans partage, l'absence de contre-pouvoirs pendant quatre

30. Revista de Estudios Europeos, n 29/30, juin 1994, La Havane.

31. Dépêche Reuters, 18 avril 1999. 
décennies, des institutions taillées sur mesure pour le Commandant en chef, une population démoralisée, La Havane quadrillée, ne préparent pas l'avenir.

\section{UNE EXPERIENCE HISTORIQUE INEDITE}

L'expérience cubaine sanctionne l'échec de l'étatisme: la collectivisation des moyens de production n'est pas à elle seule un projet politique viable parce que le seul changement de propriété ne présente aucune garantie contre la confiscation du pouvoir, que ce soit par la haute administration ou par une bureaucratie faisant un usage inefficace des richesses. Le problème posé est celui de la délégation de pouvoir ou du partage des pouvoirs, de la participation des citoyens, des salariés, des usagers impulsant un socialisme participatif que les nouvelles divisions sociales entre salariés et nouveaux travailleurs indépendants, entre blancs et noirs rendent encore plus incertain à Cuba.

L'objectif des autorités économiques est de maintenir la propriété étatique des moyens de production. Mais comment concilier gestion capitaliste des entreprises, ouverture au monde extérieur et monopole du Parti unique?

Les mesures de libéralisation prises entre 1992 et 1995 l'ont été sous la contrainte et la nécessité, la situation devant retourner à la «normale» une fois la «période spéciale» passée. Mais que signifierait un retour en arrière après la disparition du bloc soviétique ? Comment l'économie du pays va-t-elle s'adapter à la mondialisation ? A quelles conditions est-il possible d'approfondir les réformes économiques en sauvegardant le régime et le système politique en vigueur comme le souhaitent ses dirigeants ? Le système résistera-t-il au danger de se voir dépassé par des réformes économiques dont les conséquences sociales ne manqueront pas d'acquérir une dynamique propre après la disparition de son fondateur? Le vide institutionnel actuel pourrait-il être comblé par une démocratisation sociale et politique sauvegardant les acquis fondamentaux de la Révolution cubaine instaurant - expérience inédite - un socialisme rénové ? Ou la 
révolution cubaine connaîtra-t-elle le sort tragique de la première République Haïtienne ?

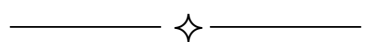

\section{Résumé}

La crise qui affecte l'île depuis presque dix ans a profondément ébranlé la société cubaine. L'adoption de réformes économiques marchandes a provoqué une dislocation idéologique et sociale profonde. Pour faire face à l'essor de la corruption et de la délinquance conséquences de la libéralisation économique, le gouvernement cubain a renforcé la répression. L'impasse politique où se trouve la direction cubaine explique ses choix autoritaires.

Mais en refusant d'accepter tout espace social autonome le pouvoir se prive des moyens démocratiques de contrebalancer les effets pervers des réformes qui ne manqueront pas d'acquérir une dynamique propre. Seule une démocratisation sociale et politique pourrait permettre de sauvegarder les acquis fondamentaux de la Révolution en évitant les périls d'une transition dangereuse.

\begin{abstract}
The crisis affecting the island for the past ten years has profoundly upset Cuban society. The adoption of commercial economic reform has provoked deep ideological and social dislocation. To face the rise of corruption and criminality, the consequence of economic liberalization, the Cuban government has strengthened its repressive practices. The political impasse that the Cuban administration finds itself in explains its choosing the path of authority. But, by refusing to accept any type of autonomous social environment, the government deprives itself of a democratic means of counterbalancing the perverse effects of reform which will ineluctably acquire their own dynamic drive. Social and political democratization alone can enable the safekeeping of the fundamental privileges of the Revolution while avoiding the risks of a dangerous transition.
\end{abstract}

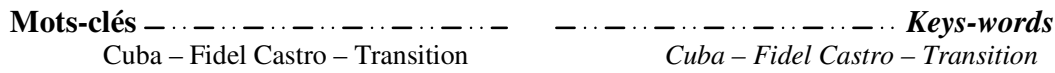

